\title{
Examining location-based and object-based components of inhibition of return in static displays
}

\author{
JIM MCAULIFFE \\ Lakehead University, Thunder Bay, Ontario, Canada \\ JAY PRATT \\ University of Toronto, Toronto, Ontario, Canada \\ and \\ CAM O'DONNELL \\ University of Western Ontario, London, Ontario, Canada
}

\begin{abstract}
Tipper and Colleagues (e.g., Jordan \& Tipper, 1998; Tipper, Driver, \& Weaver, 1991; Tipper, Weaver, Jerreat, \& Burak, 1994) have provided support for inhibition of return (IOR) being composed of a location-based and an object-based component. They were able to separate out the effects of locationbased and object-based IOR by using complex displays and displays that involved moving the cued object. The present study was designed to further examine the object- and location-based components of IOR in static displays. Three experiments were conducted that looked at the presence or absence of placeholder boxes on IOR. The first experiment was designed to replicate the results of Jordan and Tipper by presenting both objects and no-objects in the same display. In the second experiment, trials were blocked, and in the third experiment trials were presented in a random order. Overall, the results are inconsistent with the notion that independent object-based and location-based IOR components combine to produce the overall IOR effect and that additive effects are realized due to the context in which the trials are presented. We propose that a single inhibitory mechanism can account for the data.
\end{abstract}

In 1984, Posner and Cohen found that responses were slower to targets at cued locations than to targets at uncued locations if the time interval between the onset of the cue and the onset of the target was greater than $300 \mathrm{msec}$. They referred to this phenomenon as inhibition of return (IOR), indicating their original notion that attention was inhibited to return to the previously attended (i.e., cued) location. Moreover, they argued that such an inhibitory process would be extremely adaptive because it would make visual searches more efficient by biasing attention toward novel locations and away from previously inspected locations. Because of the robust nature of the effect, and the relationship to both oculomotor and attentional systems, as well as the possible role in the critical and ubiquitous need to search our visual environments, IOR has attracted a great deal of research interest over the last 2 decades (e.g., Abrams \& Dobkin, 1994; Pratt, Spalek, \& Bradshaw, 1999; Rafal, Calabresi, Brennan, \& Sciolto, 1989; Taylor \& Klein, 1998).

This research was supported by an NSERC research grant awarded to J. P. We thank Jeremy Wolfe, Steven Tipper, Lawrence Ward, and an anonymous reviewer for their helpful comments on an earlier version of this manuscript. The authors thank Marc Poirier, Eryk Przysucha, Veli Rautio, and Roy Wittock for the collection of the data. Correspondence should be sent to J. McAuliffe, School of Kinesiology, Lakehead University, 955 Oliver Road, Thunder Bay, ON, P7B 5E1, Canada (email: jim.mcauliffe@lakeheadu.ca).
In examining the possible role of IOR in search or foraging behavior, Tipper, Driver, and Weaver (1991) noted that IOR would be especially useful if attention was inhibited to return not only to previously attended static objects (such as those used by Posner \& Cohen, 1984) but also to previously attended dynamic objects. In other words, initial work on IOR indicated that it might help you find your pen on your desktop; but would it also help you find a friend moving throughout a crowded room? To examine whether IOR has an object-based component, Tipper et al. (1991) developed a procedure in which boxes presented on either side of a central fixation point moved to new spatial locations after being cued. Following the movement of the boxes, a target was presented in either the cued or the uncued box. Thus, with this procedure they were able to present targets at previously cued objects that were no longer at the previously cued locations. The results of this study indicated that IOR is object based and does move with the cued object, even if the cued box rotates $180^{\circ}$, so that it stops at the former location of the uncued box. Later, Tipper, Weaver, Jerreat, and Burak (1994) extended their method by using four boxes arranged equidistantly from the central fixation point and each rotated $90^{\circ}$ following the cuing of one of the boxes. This allowed Tipper et al. (1994) to place targets either in cued objects at new (uncued) locations or in uncued objects at cued locations. The results from this study indicated that IOR has both an object-based com- 
ponent (that moves with the cued object) and a locationbased component (that remains at the cued location) and that these two components can exist simultaneously.

The finding of two independent components of IOR has been used to explain why IOR effects in static displays tend to be much larger than either the object-based component or the location-based component of IOR found in dynamic displays. Tipper and Weaver (1998) and Weaver, Lupiáñez, and Watson (1998) have noted that the typical static IOR experiment uses placeholder boxes to indicate where the cues and targets may appear (e.g., Posner \& Cohen, 1984; Pratt et al., 1999; Rafal et al., 1989). When a cue appears at such a placeholder, both the cued object and the cued location will become inhibited. Thus, IOR found in the typical static display is a combination of both the object-based component and the location-based component and is therefore larger than the IOR found when the cued object is moved to a new location prior to the presentation of the target.

One question leading from the notion that the objectbased and location-based components of IOR summate in static displays is, what happens if there is no placeholder in which the cues and targets may occur? The prediction stemming from Tipper and Weaver (1998) and Weaver et al. (1998) is that a small IOR effect due to the location-based component should be found. This issue was examined by Jordan and Tipper (1998), who presented cues and targets in either Kanizsa apparent objects (objects formed by "Pac-man" characters arranged to form the four corners of a square) or in an objectless area (where the contours used to define the Kanizsa object were scrambled in such a way that no object was apparent). They found $42 \mathrm{msec}$ of IOR when the Kanizsa object was present and only $18 \mathrm{msec}$ of IOR when there was no apparent object, a result consistent with the notion that IOR associated with a peripheral cue has both an object-based and a location-based component.

The present study was designed to further examine the object-based and location-based components of IOR in static displays. Specifically, we wanted to (1) differentiate the two components in a static display and (2) examine the time course of the two components. The basic method of the following experiments is straightforward; on some trials placeholder boxes were present and on some trials they were absent. Thus, cues and targets could appear either in objects or in an essentially blank display. To investigate possible differences in the time course of the two components, stimulus onset asynchronies (SOAs) of 400,600 , and $800 \mathrm{msec}$ were used.

In addition to the placeholder box and SOA manipulations, on some trials a cue appeared at the fixation point following the peripheral cue and before the target, while on other trials no event occurred between the peripheral cue and the target. Over the years, fixation cues have (e.g., Abrams \& Dobkin, 1994; Kingstone \& Pratt, 1999; Tipper et al., 1991; Tipper et al., 1994) and have not been (e.g., Danzinger, Kingstone, \& Snyder, 1998; Maylor, 1985; Pratt \& McAuliffe, 1999) used in studying IOR despite the fact that little is actually known about the effect of such cues on IOR except that it may affect the time course of IOR. Specifically, attentional explanations of IOR assume that attention must be withdrawn from the cued location either by the passage of time and/or by a cue at fixation (e.g., Posner \& Cohen, 1984). If this assumption is correct, then the presence of a fixation cue should yield greater IOR at shorter SOAs, where such durations might not be sufficient for attention to be completely removed from the cued location without the help of an external event. In addition, if one of the two components (i.e., location based or object based) has a slower time course, a fixation cue may prove more beneficial to one component of IOR than the other.

\section{EXPERIMENT 1}

Jordan and Tipper (1998) found support for their twocomponent notion of object- and location-based IOR by presenting participants with both object + location and location-only cued trials within a block of trials. It is possible that the differences in IOR for objects and locations found by Jordan and Tipper were due to their experimental display and procedures. Specifically, in their study apparent objects and no-objects (i.e., locations) were presented on each trial. The present experiment was designed to conceptually replicate Jordan and Tipper using simple visual objects (i.e., placeholder boxes instead of Kanisza squares). If the presence of both objects and no-objects produced the separate effects of object- and location-based IOR found by Jordan and Tipper, then similar results should be found in the present experiment.

\section{Method}

Participants. Sixteen undergraduate students from Lakehead University volunteered to participate in the study. Each session lasted approximately $30 \mathrm{~min}$.

Apparatus and Proced ure. The experiment was conducted using a PC computer. Participants were seated at a desk in a dimly lit, sound-attenuated room. Participants were seated $38 \mathrm{~cm}$ directly in front of a computer monitor. The computer keyboard was located on the table within easy reach of the participants.

The basic trial procedure is shown in Figure 1. The trial procedures used were similar to that used by Jordan and Tipper (1998). The main difference is that objects were placeholder boxes rather than apparent objects. For all trials, each trial began with a blank screen for $1,000 \mathrm{msec}$. Following that, a central fixation dot and two boxes, aligned on either the horizontal or vertical meridian, were presented on the monitor. The central fixation dot served as the fixation location. The boxes were centered $5.5^{\circ}$ from the middle of the center fixation dot and were $1^{\circ}$ square. After the initial display was presented for $1,000 \mathrm{msec}$, a cue consisting of a white unfilled square $\left(.50^{\circ}\right.$ square $)$ centered $5.5^{\circ}$ above, below, to the left, or to the right of the fixation box was presented for $200 \mathrm{msec}$ and then removed. The cues did not reliably predict target location. There was a delay for $200 \mathrm{msec}$ and then the central fixation dot was expanded slightly for $200 \mathrm{msec}$. Finally, the target was presented following a delay of $400 \mathrm{msec}$. The target consisted of a filled in square $\left(.50^{\circ}\right.$ square) and was centered $5.5^{\circ}$ above, below, to the left, or to the right of the fixation dot. The target remained on until the participant responded or $1,500 \mathrm{msec}$ elapsed. The SOA was $1,000 \mathrm{msec}$. The intertrial interval was $1,500 \mathrm{msec}$.

The target and cue appeared equally often in each of the four locations. Trials in which the cue appeared in a placeholder box 


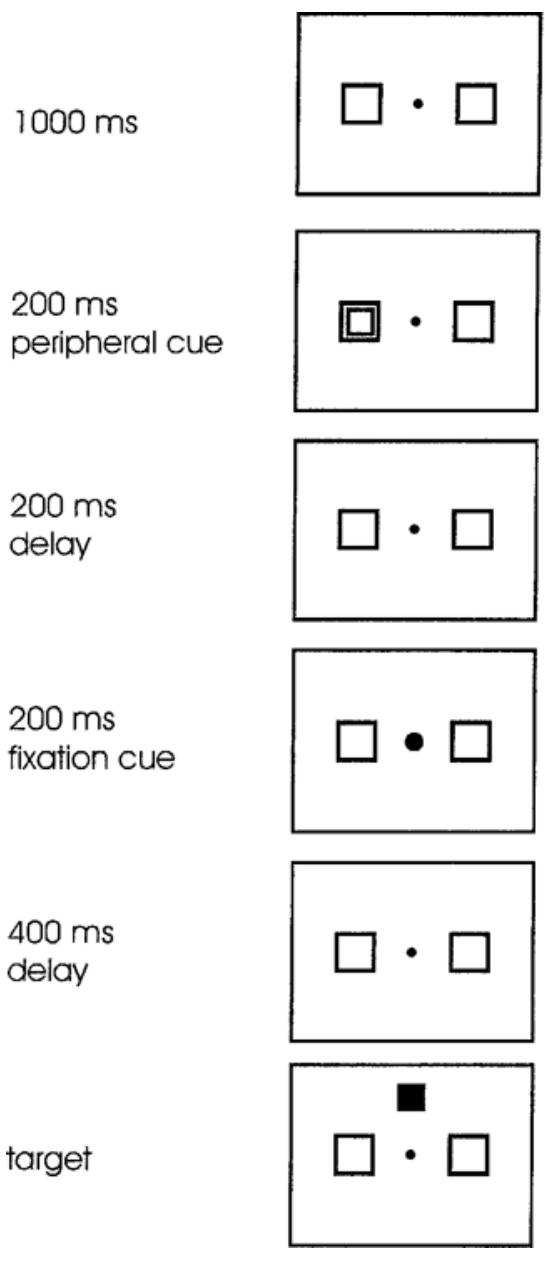

Figure 1. The basic trial sequence used in Experiment 1. The cues and targets were equally likely to appear above, below, to the left, or to the right of fixation. The two placeholders were also equally likely to be horizontally or vertically aligned. See the text for details.

and the target appeared in a placeholder box were considered to be placeholder-present trials. Similarly, when the cue and target appeared in the locations not defined by a placeholder box (empty space), the trial was considered to be a placeholder-absent trial. In addition, to complete the trial sequence, trials in which either (1) the cue was presented in a placeholder box and the target was presented in empty space or (2) the cue was presented in empty space and the target was located within a placeholder box were included. Consistent with Jordan and Tipper (1998), the filler trials were included to insure that the target location could not be predicted on the basis of cue location.

Participants were instructed to remain fixated on the center fixation point for the duration of each trial and to respond to the appearance of the target by pressing the spacebar as quickly and accurately as possible. ${ }^{1}$ Catch trials, in which no response was to be made, were included on $20 \%$ of the trials and they consisted of all the events in the trial sequence except for the presentation of the target. The participants were instructed not to respond on such trials. A short error tone was sounded if the participant responded too quickly (less than $100 \mathrm{msec}$ ), too slowly (greater than 1,000 msec), or on a catch trial.

Design. Sixteen participants completed two blocks of 200 trials. Each block of 200 trials consisted of 40 placeholder-present $(20$ cued and 20 uncued) trials, 40 placeholder-absent (20 cued and 20 uncued) trials, 80 filler trials, and 40 catch trials. The filler trials were not submitted to further analysis. For all trials, the presentation of the cues and targets was equally likely above, below, to the left, and to the right of the fixation dot. A short break was provided every 50 trials in each block of trials and at the end of the first block of trials.

\section{Results and Discussion}

The mean reaction times (RTs) from the errorless trials are shown in Table 1. As in Jordan and Tipper (1998), the data were analyzed with a 2 (type of stimuli: placeholder present, placeholder absent) $\times 2$ (trial type: cued or uncued) repeated measures analysis of variance (ANOVA). The analysis revealed that responses on cued trials $(333 \mathrm{msec})$ were significantly slower than responses on uncued trials $\left[300 \mathrm{msec} ; F(1,15)=52.9, M S_{\mathrm{e}}=337\right.$, $p<.001]$, indicating the typical IOR effect. In addition, the main effect of type of stimuli (placeholder present $=$ $321 \mathrm{msec}$, placeholder absent $=312 \mathrm{msec}$ ) was significant $\left[F(1,15)=5.9, M S_{\mathrm{e}}=218, p<.03\right]$. More importantly, the type of stimuli $\times$ trial type interaction was significant $\left[F(1,15)=5.0, M S_{\mathrm{e}}=90.5, p<.05\right]$. Post hoc Scheffé tests were conducted to determine the nature of the interaction. There was greater IOR in the placeholderpresent trials $(39 \mathrm{msec})$ than in the placeholder-absent trials $(28 \mathrm{msec})$.

Overall, errors were made on $1 \%$ of the trials. Three types of errors were possible: false positives (responses on catch trials), responding too fast (RTs less than $100 \mathrm{msec}$ ), and responding too slow (RTs greater than $1,000 \mathrm{msec}$ ). On average, participants committed 1.5 false positive errors per session, which represents $1.9 \%$ of the catch trials ( 80 trials). In addition, participants averaged 2.0 too fast errors and .5 too slow errors per session, which represents less than $1 \%$ of the total targetpresent trials.

The findings of the present experiment are consistent with those of Jordan and Tipper (1998). Jordan and Tipper found greater IOR $(42 \mathrm{msec})$ in the apparent-object condition (Kanisza squares) than in the no-apparentobject condition $(18 \mathrm{msec})$. The same basic pattern was found in the present experiment with more IOR in objects (placeholder present, $39 \mathrm{msec}$ ) than in locations (placeholder absent, $28 \mathrm{msec}$ ), although the difference between the two conditions was less than that observed by Jordan and Tipper (Figure 2). This may have been due to the relatively complex display used by Jordan and Tipper and the relatively sparse display used in the present experiment.

Table 1

The Mean Cued and Uncued Reaction Times (in Milliseconds) for Experiment 1

(Objects and No-Objects Present in the Same Display)

\begin{tabular}{ccc}
\hline Cued Object & Uncued Object & IOR \\
340 & 301 & 39 \\
Cued No-Object & Uncued No-Object & IOR \\
326 & 298 & 28 \\
\hline
\end{tabular}

Note-IOR, inhibition of return. 


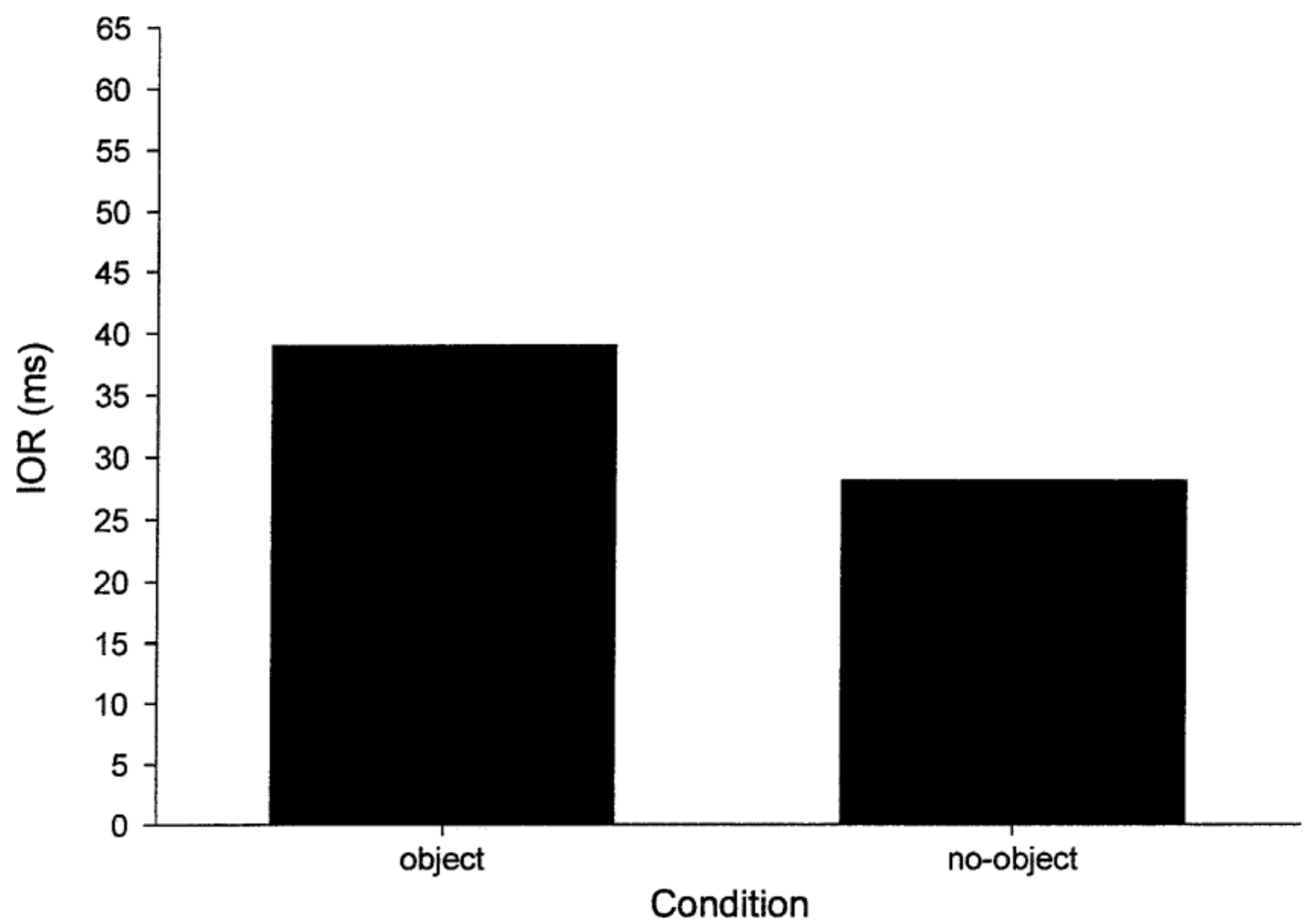

Figure 2. Mean inhibition of return (IOR) effects (cued reaction time [RT] minus uncued RT) for the conditions in Experiment 1.

\section{EXPERIMENT 2}

The present experiment more thoroughly examined object-based and location-based IOR by manipulating the placeholder boxes (present or absent), SOA $(400,600$, $800 \mathrm{msec}$ ), and the fixation cue (present or absent). In this experiment, the SOA manipulation was between groups, while the placeholder box and fixation cue manipulations were within groups. The within-groups manipulations were blocked so that the participants knew what type of display and what trial events would be presented within a block of trials.

Stemming from the work of Jordan and Tipper (1998), Tipper and Weaver (1998), Weaver et al. (1998), and Experiment 1 of the present study, the prediction was that a larger IOR effect would be found with the placeholder present (object-based IOR plus location-based IOR) than when the placeholders were absent (only location-based IOR). Predictions were also made about the fixation cues and SOA interaction. An attentional explanation of IOR would predict that at very short SOAs, the presence of a fixation cue would result in larger inhibitory effects, although the effect of this interaction on the two components is uncertain.

\section{Method}

Participants. Forty-eight undergraduate students from Lakehead University, separated randomly into three groups of 16 people each, participated in the experiment. Each experimental session lasted approximately $30 \mathrm{~min}$ and each person received course credit for his/her participation.

Apparatus and Procedure. The experiment was conducted using a PC computer. Participants were seated at a desk in a dimly lit, sound-attenuated room. Participants were seated $38 \mathrm{~cm}$ directly in front of a computer monitor. The computer keyboard was located on the table within easy reach of the participants.

The basic trial sequence is shown in Figure 3. For all trials, each trial began with a blank screen for $1,000 \mathrm{msec}$. Following that, the initial display in a trial sequence differed depending on whether or not placeholder boxes were present on a trial. On placeholderpresent trials, a central fixation dot and a peripheral box on either side of the fixation dot were presented. The boxes were centered $5.5^{\circ}$ from the fixation dot and were $1^{\circ}$ square. On placeholderabsent trials, only the central fixation point was presented. After the initial display was presented for $1,000 \mathrm{msec}$, a cue consisting of a filled in circle $\left(.90^{\circ}\right.$ in diameter) appeared centered $5.5^{\circ}$ to the left or the right of the fixation cue, for both placeholder-present and placeholder-absent trials. The cue was presented for $200 \mathrm{msec}$ and then removed. The cues were not predictive of target location. On fixation-cue-present trials in the 400-msec SOA condition, the fixation cue expanded slightly for $200 \mathrm{msec}$ immediately after the offset of the cue and then the target was presented. On fixation-cuepresent trials in the 600-msec SOA condition, there was a delay of $100 \mathrm{msec}$ after the offset of the peripheral cue, then the fixation cue expanded slightly for $200 \mathrm{msec}$, another delay of $100 \mathrm{msec}$, and then the target was presented. On fixation-cue-present trials in the $800-\mathrm{msec}$ SOA condition, there was a delay of $200 \mathrm{msec}$ after the offset of the cue, then the fixation cue expanded slightly for $200 \mathrm{msec}$, another delay of $200 \mathrm{msec}$, and then the target was presented. The presence of the fixation cue served as the reorienting event. On 


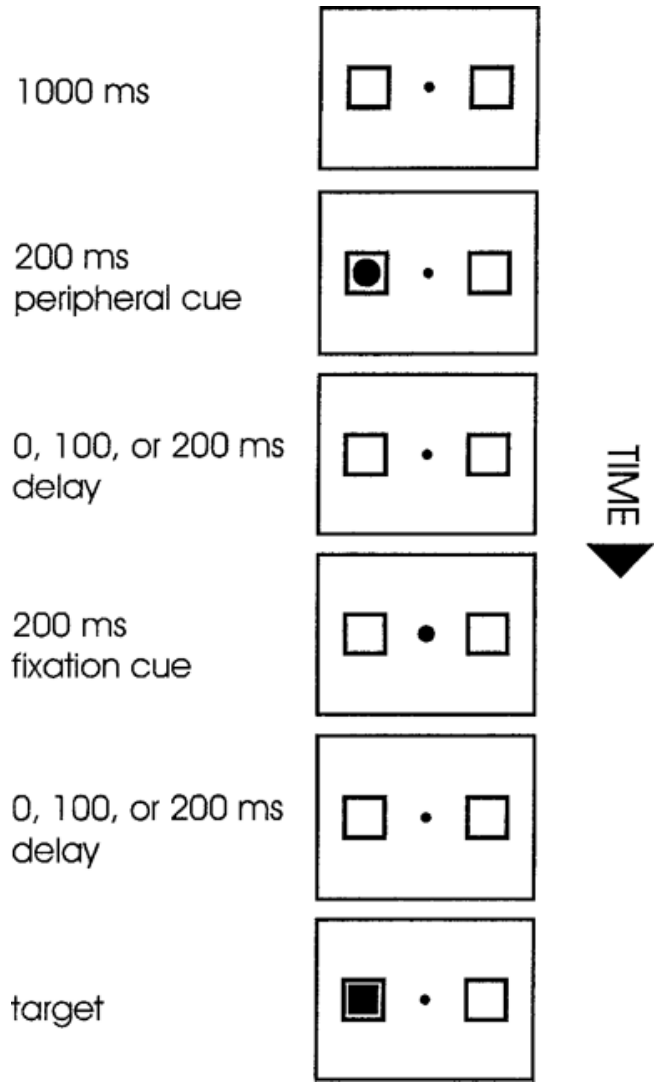

Figure 3. The basic trial sequence used in Experiments 2 and 3. The cues and targets were equally likely to appear to the left or right of fixation, and the fixation cue and the placeholder boxes were equally likely to be present or absent. See the text for details regarding the duration of the delays.

fixation-cue-absent trials, there was a delay of 200,400 , or $600 \mathrm{msec}$ after the offset of the cue and before the target was presented, depending on the SOA condition (i.e., 400-, 600-, or 800-msec SOAs). The target consisted of a filled in square $\left(.90^{\circ}\right.$ square $)$ and was centered $5.5^{\circ}$ to the left or right of the fixation point. Thus, the target could appear in the cued location or the uncued location. The target remained present until a response had been made or $1,000 \mathrm{msec}$ had elapsed.

Participants were instructed to remain fixated on the center fixation point for the duration of each trial and to respond to the appearance of the target by pressing the spacebar as quickly and accurately as possible. Catch trials, in which no response was to be made, were presented on $20 \%$ of the trials and they consisted of all the events in the trial sequence except for the presentation of the target. The participants were instructed not to respond on such trials. A short error tone was sounded if the participant responded too quickly (less than $100 \mathrm{msec}$ ), too slowly (greater than 1,000 msec), or if they responded on a catch trial.

Design. Sixteen participants were randomly assigned to each of the three SOA conditions $(400,600$, or $800 \mathrm{msec}$ ). Each participant completed four blocks of 60 trials (48 test trials and 12 catch trials). The four trial blocks were as follows: placeholder boxes present and fixation cue present, placeholder boxes present and fixation cue absent, placeholder boxes absent and fixation cue present, placeholder boxes absent and fixation cue absent. The presentation of the trial blocks was counterbalanced using a latin square. For all trials, the presentation of the cues and targets was equally likely at both the left and right locations. A short break was provided at the end of each block.

\section{Results and Discussion}

The mean RTs from the correct trials appear in Table 2 and were analyzed with a 2 (trial type: cued or uncued) $\times 2$ (placeholder: present or absent) $\times 2$ (fixation cue: present or absent) $\times 3$ (SOA: 400,600 , or $800 \mathrm{msec})$ ANOVA. The analysis revealed that RTs on cued trials (339 msec) were significantly slower than RTs on uncued trials $\left[302 \mathrm{msec}, F(1,45)=183, M S_{\mathrm{e}}=132,388\right.$, $p<.0001]$. This is the typical IOR effect. In addition, the main effect for placeholder (present $=324 \mathrm{msec}$, absent $=316 \mathrm{msec})$ was significant $[F(1,45)=6.89$, $\left.M S_{\mathrm{e}}=915, p<.01\right]$. However, trial type did not interact with placeholder on any level, indicating that IOR was not affected by the presence or absence of placeholder boxes. The main effect of fixation cue was not significant. Two of the two-way interactions were significant: the SOA $\times$ fixation interaction $\left[F(1,45)=6.13, M S_{\mathrm{e}}=\right.$ $1,294, p<.01]$ and the trial type $\times$ fixation interaction $\left[F(1,45)=11.21, M S_{\mathrm{e}}=474, p<.001\right]$. In addition, the three-way interaction between SOA, trial type, and fixation was also significant $\left[F(2,45)=4.22, M S_{\mathrm{e}}=474, p<\right.$ $.05]$. No other interactions reached significance.

To further determine the effect of placeholder boxes and the fixation cue on IOR, separate two-way ANOVAs (placeholder $\times$ fixation cue) were calculated on the IOR effects (cued RT minus uncued RT) for each SOA condition. The IOR effects are shown in Figure 4. All main effects and interactions involving placeholder boxes were not significant $(p>.05)$. The only significant effect found was for the fixation cue in the 400-msec SOA condition $\left[F(1,15)=15.92, M S_{\mathrm{e}}=989, p<.001\right]$, with more IOR (48 msec) when the fixation cue was present than when it was absent (16 msec).

Overall, errors were made on $1.6 \%$ of the trials. As in the previous experiment, three types of errors were possible: false positives (responses on catch trials), responding too fast (RTs less than $100 \mathrm{msec}$ ), and responding too slow (RTs greater than $1,000 \mathrm{msec}$ ). On average, participants committed 2.6 false positive errors per session,

Table 2

The Mean Cued and Uncued Reaction Times (RTs, in Milliseconds) for Experiment 2 (Blocked Presentation)

\begin{tabular}{lllccc}
\hline SOA & Placeholder & Fixation Cue & Cued RT & Uncued RT & IOR \\
\hline 400 & present & present & 367 & 319 & 48 \\
400 & absent & present & 350 & 304 & 46 \\
400 & present & absent & 324 & 309 & 15 \\
400 & absent & absent & 324 & 307 & 17 \\
600 & present & present & 351 & 307 & 44 \\
600 & absent & present & 341 & 296 & 45 \\
600 & present & absent & 334 & 307 & 27 \\
600 & absent & absent & 329 & 293 & 36 \\
800 & present & present & 333 & 291 & 42 \\
800 & absent & present & 326 & 287 & 39 \\
800 & present & absent & 344 & 305 & 39 \\
800 & absent & absent & 340 & 297 & 43 \\
\hline
\end{tabular}

Note-SOA, stimulus onset asynchrony; IOR, inhibition of return. 


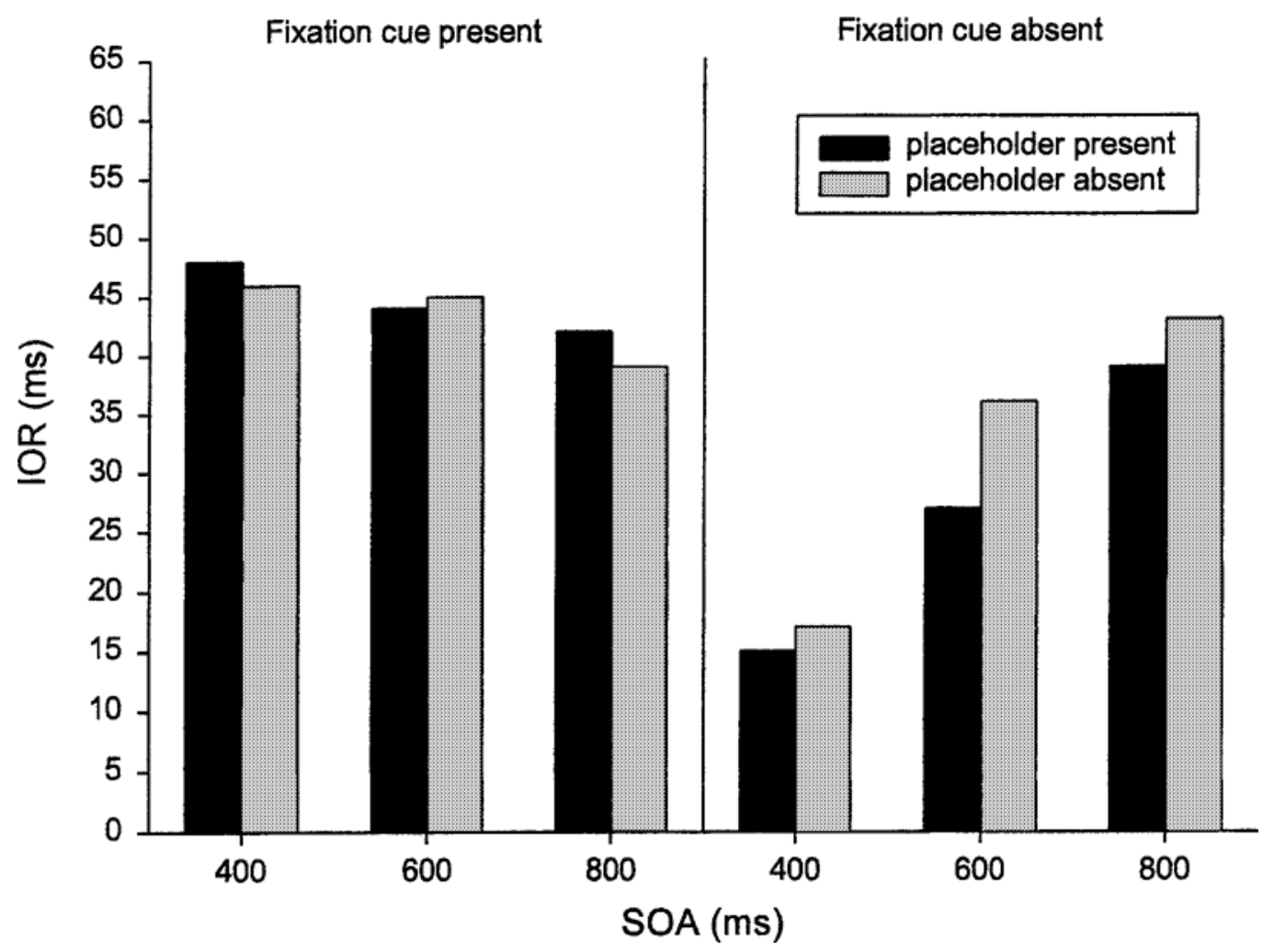

Figure 4. Mean inhibition of return (IOR) effects (cued reaction time [RT] minus uncued RT) for the conditions in Experiment 2 (blocked presentation). SOA, stimulus onset asynchrony.

which represents $4.3 \%$ of the catch trials (60 trials). In addition, participants averaged 1.8 too fast errors and .5 too slow errors per session, which represents $1.0 \%$ of the total target-present trials.

If object- and location-based IOR are additive components of the IOR phenomenon, then the presence of placeholder boxes on any given trial should produce more IOR. Overall, the presence and absence of the placeholder boxes had virtually no effect on IOR at any SOA. In other words, the magnitude of the IOR effect was not diminished when objects were not present in the view field. This finding is inconsistent with the predictions stemming from Jordan and Tipper (1998), Tipper and Weaver (1998), and Weaver et al. (1998). Because separate object-based and location-based effects were not found in these static displays, the SOA manipulation provided no information about possible time course differences with these components.

The only effect found in the first experiment was that the presence of the fixation cue produced greater IOR at the 400-msec SOA than did the absence of the fixation cue. This finding is consistent with the notion that attention needs to be removed from the peripherally cued location to produce IOR. Without the fixation cue at the 400 -msec SOA, attention may not have been totally disengaged from the cued location and thus a smaller IOR was found. However, the attentional capturing fixation cue may have allowed attention to be disengaged and moved from the cued location, producing a much larger IOR effect. The lack of any effect at the 600- and 800-msec SOAs suggests that those durations were sufficient for attention to be disengaged and moved to (presumably) the fixation location without the aid of the fixation cue.

\section{EXPERIMENT 3}

One possible reason for lack of effects found with the placeholder manipulation in the second experiment was the blocked design, which provided for a degree of certainty for a given set of trials. However, IOR experiments typically randomize the presentation of the manipulated variables (e.g., Danzinger et al., 1998; Pratt, Adam, \& McAuliffe, 1998; Rafal et al., 1989). Such was the case with Jordan and Tipper (1998) and Experiment 1 of the present study: A decrease in IOR was found when there were no objects apparent in the display. In these studies, the presence or absence of objects was equally probable on any given trial. To examine the possibility that the blocking of trials contributed to the findings of the previous experiment, the present experiment used the same trial sequences as Experiment 2 but used a random trial presentation order. 


\section{Method}

Participants. Forty-five undergraduate students from Lakehead University, separated randomly into three groups of 15 people each, participated in the experiment. Each experimental session lasted approximately $30 \mathrm{~min}$ and each person received course credit for his/her participation.

Apparatus and Procedure. The apparatus and procedure used were exactly the same as those used in the previous experiment.

Design. The design was the same as that of Experiment 2 except that any of the four conditions (placeholder boxes present and fixation cue present, placeholder boxes present and fixation cue absent, placeholder boxes absent and fixation cue present, placeholder boxes absent and fixation cue absent) were equally likely to occur within each block of 60 trials.

\section{Results and Discussion}

The mean RTs from the errorless trials are shown in Table 3 and were analyzed using a 2 (trial type) $\times 2$ (placeholder) $\times 2$ (fixation cue) $\times 3$ (SOA) ANOVA. The analysis revealed that responses on cued trials $(374 \mathrm{msec})$ were significantly slower than responses on uncued trials [332 msec; $\left.F(1,42)=109, M S_{\mathrm{e}}=1,438, p<.0001\right]$, indicating the typical IOR effect. As in Experiment 2, the main analyses concerning the main purpose of the study deal with the placeholder factor. The only significant effect involving the placeholder factor was the trial type $\times$ placeholder interaction $\left[F(1,42)=5.87, M S_{\mathrm{e}}=\right.$ $339, p<.01]$. In addition, the main effect of fixation (present $=339 \mathrm{msec}$, absent $=367 \mathrm{msec}$ ) was significant $\left[F(1,42)=79.53, M S_{\mathrm{e}}=902, p<.0001\right]$. Three of the two-way interactions were significant: SOA $\times$ fixation cue $\left[F(1,42)=11.36, M S_{\mathrm{e}}=902, p<.0001\right]$, trial type $\times$ fixation cue $\left[F(1,42)=9.84, M S_{\mathrm{e}}=333, p<.01\right]$. The three-way interaction between SOA, trial type, and fixation was also significant $\left[F(2,42)=3.42, M S_{\mathrm{e}}=333, p<\right.$ .05]. No other main effects or interactions were significant.

As was done previously, the effect of the fixation cue and placeholder boxes on IOR was determined by separate two-way ANOVAs (placeholder $\times$ fixation cue) calculated on the IOR effects for each SOA. The IOR effects are shown in Figure 5. In the 400-msec SOA condition, there was a significant main effect of placeholder $[F(1,14)=$

Table 3

The Mean Cued and Uncued Reaction Times (RTs, in Milliseconds) for Experiment 3 (Random Presentation)

\begin{tabular}{lllccc}
\hline SOA & Placeholder & Fixation Cue & Cued RT & Uncued RT & IOR \\
\hline 400 & present & present & 371 & 313 & 58 \\
400 & absent & present & 359 & 326 & 33 \\
400 & present & absent & 362 & 330 & 32 \\
400 & absent & absent & 362 & 346 & 16 \\
600 & present & present & 358 & 309 & 49 \\
600 & absent & present & 352 & 306 & 46 \\
600 & present & absent & 386 & 349 & 37 \\
600 & absent & absent & 374 & 348 & 26 \\
800 & present & present & 374 & 323 & 51 \\
800 & absent & present & 362 & 312 & 50 \\
800 & present & absent & 416 & 363 & 53 \\
800 & absent & absent & 410 & 358 & 52 \\
\hline
\end{tabular}

Note-SOA, stimulus onset asynchrony; IOR, inhibition of return. $\left.15.49, M S_{\mathrm{e}}=403, p<.001\right]$. The magnitude of IOR was greater when the placeholder was present $(45 \mathrm{msec})$ than when it was absent $(25 \mathrm{msec})$. The interaction of placeholder and fixation cue in the 400-msec SOA condition was not significant $\left[F(1,14)=.61, M S_{\mathrm{e}}=410, p=\right.$ $.45]$. The main effects of placeholder in the $800-\mathrm{msec}$ SOA condition $\left[F(1,14)=.75, M S_{\mathrm{e}}=1,068, p=.40\right]$ and the $600-\mathrm{msec}$ SOA condition $[F(1,14)=.007$, $\left.M S_{\mathrm{e}}=564, p=.93\right]$ were nonsignificant. In addition, the placeholder $\times$ fixation cue interactions in the 800msec SOA condition $\left[F(1,14)=.004, M S_{\mathrm{e}}=466, p=\right.$ $.95]$ and the 600-msec SOA condition $[F(1,14)=.38$, $\left.M S_{\mathrm{e}}=384, p=.55\right]$ were not significant.

A secondary purpose of the study was to look at the effect of the fixation cue. In the 400-msec SOA condition there was a significant main effect of fixation cue $\left[F(1,14)=39.37, M S_{\mathrm{e}}=177, p<.0001\right]$. As in the previous experiment, the magnitude of the IOR was greater when the fixation cue was present $(46 \mathrm{msec})$ than when it was absent $(24 \mathrm{msec})$. The interaction of placeholder and fixation cue in the 400-msec SOA condition was not significant $\left[F(1,14)=.61, M S_{\mathrm{e}}=410, p=.45\right]$. There was also a significant main effect of fixation in the 600msec SOA condition $\left[F(1,14)=11.5, M S_{\mathrm{e}}=358, p<\right.$ $.01]$, with greater IOR with the fixation cue $(48 \mathrm{msec})$ than without the fixation cue $(31 \mathrm{msec})$. The main effect of fixation cue in the 800-msec SOA condition failed to reach significance $\left[F(1,14)=.036, M S_{\mathrm{e}}=1,466, p=.85\right]$.

Overall, errors were made on $1.7 \%$ of the trials. As in Experiments 1 and 2, three types of errors were possible: false positives (responses on catch trials), responding too fast (RTs less than $100 \mathrm{msec}$ ), and responding too slow (RTs greater than $1,000 \mathrm{msec}$ ). On average, participants committed 1.8 false positive errors per session, which represents $3.0 \%$ of the catch trials (60 trials). In addition, participants averaged 2.2 too fast errors and 1.1 too slow errors per session, which represents less than $1.5 \%$ of the total target-present trials.

The most interesting finding from Experiment 3 of this study is that the presence of placeholder boxes resulted in greater IOR at the shortest SOA $(400 \mathrm{msec})$. This is consistent with the two-component notion of Jordan and Tipper (1998), Tipper and Weaver (1998), and Weaver et al. (1998). However, the effect disappeared at the 600and $800-$ msec SOAs. The implications of this finding will be discussed in the General Discussion section.

Similar to the findings from Experiment 2, the presence of a fixation cue resulted in a larger magnitude of IOR at the 400-msec SOA. In addition, with the random trial order, this effect persisted in the 600-msec SOA but disappeared by the $800-\mathrm{msec}$ SOA. This finding further supports the notion that IOR is, to some extent, an attentional process and is affected by the orienting of attention away from the peripherally cued location. Further, the time course of any volitional disengagement and movement of attention from the cued location to the fixation location (when no fixation cue is present) appears to be 


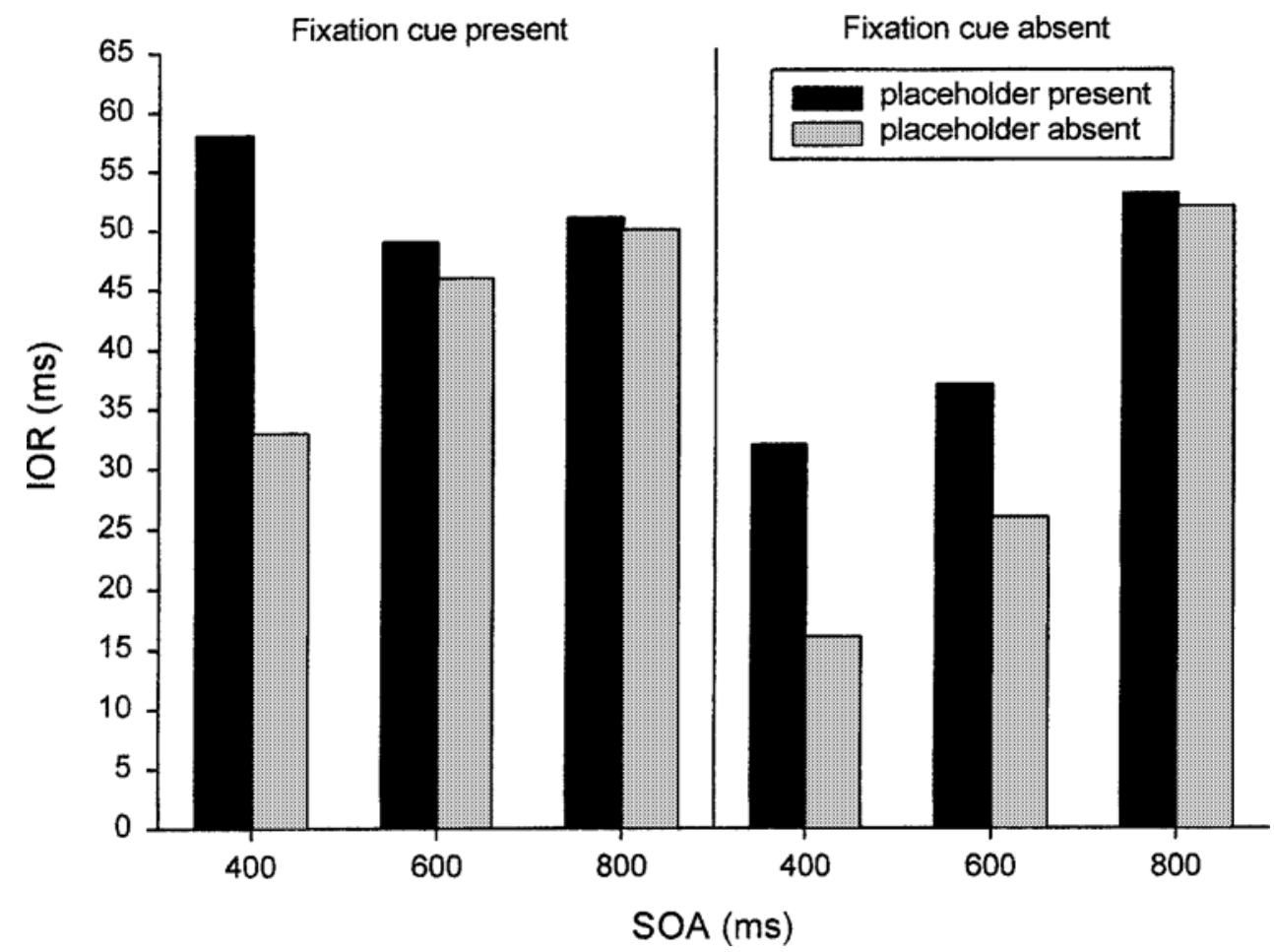

Figure 5. Mean inhibition of return (IOR) effects (cued reaction time [RT] minus uncued RT) for the conditions in Experiment 3 (random presentation). SOA, stimulus onset asynchrony.

slower than when the presence or absence of a fixation cue is predictable.

\section{GENERAL DISCUSSION}

Previous research on IOR using dynamic displays, in which the cued object is moved to a new location prior to the presentation of the target, has indicated that the inhibition occurs both at the original location of the cue and at the cued object (e.g., Abrams \& Dobkin, 1994; Tipper et al., 1991; Tipper et al., 1994). These findings have led Tipper and colleagues (e.g., Tipper et al., 1991; Tipper et al., 1994) to conclude that IOR consists of two separate components, a location-based component and an object-based component. Recently, Tipper and Weaver (1998) and Weaver et al. (1998) have suggested that these two separate components contribute to the overall inhibitory effect found in static displays. They noted that the typical IOR paradigm uses a static display with placeholder boxes that indicate the possible locations of the cues and targets. However, the placeholders are objects in the display and thus the IOR found in such experimental situations consists of the summation of both location-based IOR and object-based IOR. Support for this hypothesis comes from Jordan and Tipper (1998), who found more IOR when cues and targets appeared in apparent objects (Kanizsa squares) than when the cues and targets occurred at locations without any apparent object. The greater IOR with the apparent objects was thought to reflect the contribution of object-based IOR over and above the location-based IOR effect. The results from the present study, however, suggest an alternative conceptualization of IOR to the two-component model.

In the present study, a static display was used in which the placeholder boxes were either present or absent. Note that unlike the objects in the Jordan and Tipper (1998) study, the objects in the present study did not require modal completion but rather were directly visible to the participants. The prediction stemming from Jordan and Tipper, Tipper and Weaver (1998), and Weaver et al. (1998) is quite clear: More IOR should be found when the placeholders are present (location-based IOR plus object-based IOR) than when the placeholders are absent (location-based IOR only). In Experiments 2 and 3, there were 12 opportunities to observe any differences in IOR between placeholder-present and placeholder-absent conditions ( 2 trial presentation orders $\times 2$ fixation cue conditions $\times 3$ SOAs). In 10 of 12 conditions, no differences in IOR were found between when the placeholders were present and when they were absent. Moreover, not only were the differences in IOR between present and absent placeholders not statistically different, but as can be seen by Figures 4 and 5, the magnitude of the IOR effects with and without placeholders was almost identical in several of the conditions. The only conditions in which more IOR was found with placeholders present than absent was when the presentation order was random and the SOA was $400 \mathrm{msec}$. 
Although the results from Experiments 2 and 3 indicated that similar magnitudes of IOR can be found with and without placeholders, this is not always the case. More specifically, the results from the first experiment replicate the results of Jordan and Tipper (1998) using the same basic stimuli as were used in Experiments 2 and 3. The results from Experiment 1 were consistent with Jordan and Tipper since greater IOR was found when cues and targets were presented within the placeholder boxes (i.e., objects) than when they were presented in empty space (i.e., no-objects). Two key points can be made about the results of the first experiment. First, the greater IOR obtained in the apparent-object condition in Jordan and Tipper's experiment was not due to the nature of the stimuli used, since similar results were obtained with a relatively simple display. Jordan and Tipper cleverly used Kanisza squares to form objects, thus reducing the differences between objects and no-objects in their display. However, the nature of the display does not seem to be the critical factor in realizing a separation between objectand location-based IOR effects, since we were able to replicate their results using placeholder boxes as our object trials and empty space as our no-object trials. Further, since the stimuli in Experiment 1 were similar to those used in Experiments 2 and 3, the findings in the last two experiments cannot be attributed to stimuli used. Second, taken together with the results of Experiment 2 and 3 , the greater IOR in the placeholder-present trials may be attributed to the context in which the trials were presented. Thus, only when the visual display includes possible target locations at empty locations and placeholder locations will differential IOR effects be found.

Overall, the results from the present study are largely inconsistent with the notion that object-based and locationbased IOR components combine to produce an overall IOR effect in static displays when placeholders are present. One possible caveat to keep the two-component model viable would be to suggest that there is some limit to the amount of IOR that can be directly at a specific object or location. When objects and locations appear in the same display, the object + location IOR approaches that upper limit. However, when objects and locations appear in separate displays, then the location IOR effect can also approach the maximum limit of IOR (i.e., samemagnitude IOR effects for location and object + location conditions). Although keeping the two-component notion without additivity is possible, it's not clear what might cause the additivity to be present or absent.

Rather, the findings from the present study may be better accounted for by a single-component IOR mechanism that treats objects and locations differently. Such a single mechanism requires a few assumptions. The first assumption is that there is an upper limit to the amount of IOR that can be applied to any given object or location (i.e., there is a limited amount of IOR). The second assumption is that a cued location will get as much inhibition as is possible at that moment. The third assumption is that when searching a scene, people are more likely to orient their attention toward an object in the visual field than in an empty space. Indeed, there is considerable evidence to suggest that the visual attention system is especially sensitive to objects (e.g., Yantis \& Hillstrom, 1994). Thus, when there are only objects or locations in the visual display, the maximal amount of IOR will be applied to the cued location or cued object. However, when there are objects and empty locations in the same display, the objects will receive more IOR because attention is more likely to be directed to an object than an empty space.

With the assumption that there is a maximum limit to the amount of IOR, the notion that IOR consists of a single inhibitory mechanism that affects both cued locations and objects can account for much of the object-based IOR literature. For example, assume that in a static display using a fixation cue, a long SOA, and a detection target, an upper limit of $40 \mathrm{msec}$ of IOR will be found. This 40 msec of IOR will be found regardless of whether or not placeholders are present (as found in the present study). If, however, the same experiment was conducted with a dynamic display, smaller and roughly equivalent magnitudes of IOR would be expected to be found for both the cued object at a new location and the cued location (e.g., Tipper et al., 1994). The two-component notion suggests that this occurs because the IOR produced by each component is approximately equal. The singlecomponent notion suggests that the location where the object was cued is as viable a search location as the object itself because an object was cued at that location, not just an empty space.

At the moment, it is possible to tentatively identify two situations, based on the available research, in which an object in the visual field is likely to show more IOR than a location. The findings of the present experiments and Jordan and Tipper (1998) indicate that the visual context in which the objects and/or locations occur is sufficient to produce separate effects. On the one hand, when only objects or locations are presented, they will be attended in similar manners and the result will be a similar amount of IOR (Experiments 2 and 3). On the other hand, when objects and locations occur, there is a bias to attend to the objects over locations, and this results in greater IOR for the attended object (Experiment 1 and Jordan and Tipper). The second situation occurs at very short SOAs, where once again the bias to attend to objects may allow for larger IOR effects to be produced earlier at cued objects (Experiments 2 and 3). Once enough time has passed from the presentation of the cue, both objects and locations are inhibited in similar manners (Experiment 2 and 3).

In suggesting that there is a single component to IOR that affects both locations and objects, we have outlined only two situations (visual context and short SOAs) in which more IOR should be found for objects over locations. There may be more situations in which the presence of an object in the visual field increases IOR, but there have been only a limited number of studies that have examined IOR effects between locations and objects. In addition, there are situations in which objects might produce less IOR. For example, Tipper, Jordan, and Weaver 
(1999) found that when three dynamic moving objects were linked together with connecting lines, the previously found inhibition at the cued location (after the cued object had moved to a new location) was replaced by facilitation. Clearly, more research will be required before definitive statements about what situations will produce greater or less IOR with objects can be made.

An important assumption regarding the notion that IOR does not consist of separate location-based and object-based components, but rather is a unitary phenomenon, is that under some situations objects will be more effectively attended to and this will result in greater IOR. This assumption clearly implicates the attentional system as the locus of the inhibitory effect. Indeed, the notion that IOR occurs because attention is inhibited to return to previously attended locations has consistently received support over the last 2 decades (e.g., Chasteen \& Pratt, 1999; Maylor, 1985; Tipper et al., 1991; Tipper et al., 1994). However, a motor explanation for IOR has recently been advanced (Klein \& Taylor, 1994; Taylor \& Klein, 1998) in which IOR is thought to inhibit responses. Of special interest to the location/object IOR separation is a finding from Abrams and Dobkin (1994; see also Abrams \& Pratt, 2000) that the IOR that inhibits eye movements occurs for cued locations but not for cued objects. Our conception of IOR, following Kingstone and Pratt (1999), is that IOR exists in both the attentional and the oculomotor systems. In regard to the issue at hand, attentional IOR inhibits returning attention to both cued locations and objects while oculomotor IOR inhibits eye movements only to cued locations.

Although the issue of IOR to locations and objects was the primary focus of the present study, it also provides some information regarding the role of the fixation cue in IOR. In general, the present results indicate that larger IOR effects at the shorter SOAs were found when the fixation cues were present. As noted earlier, this is consistent with the notion that IOR is an attentional phenomenon and that attention must be disengaged and moved from the peripherally cued location in order for IOR to occur. It should be noted that had we used shorter SOAs (less than $200 \mathrm{msec}$ ), we would expect that the IOR effect would have been completely removed in the absence of the fixation cue, but due to the cuing procedure we used, the shortest SOA possible was $400 \mathrm{msec}$. However, Wright and Richard (1996) did find IOR at a $200-m s e c$ SOA with fixation cues. The present findings regarding the fixation cue also indicate that researchers studying IOR using static displays and typical long SOAs (500 msec-1,200 msec) should not be concerned with the presence and absence of fixation cues. It appears that given sufficient time, experimental designs using or not using fixation cues produce equivalent IOR effects.

In summary, the major conclusion from the present study is that the results are somewhat inconsistent with the notion of separate object-based and location-based components of IOR. Rather, we propose that a unitary attentional IOR inhibits the return of attention to both lo- cations and objects, with greater IOR occurring for cued objects in some situations. The nature of the situations in static displays where greater IOR is realized for a cued object is limited to situations of some uncertainty (i.e., random trial order), some time constraint (short SOAs), and in displays where both objects and no-objects are present in the same display. Further research is necessary to determine the exact properties of an object that would result in that object promoting greater IOR effects. Of secondary interest in this study was the role of the fixation cue on IOR. Although this is mainly a methodologicalconcern, it is worth noting that the presence of a fixation cue facilitated the return of attention at the shorter SOAs, but did not interact with the presence or absence of placeholder boxes. As a result the interpretation of the role of the placeholder boxes on IOR was fairly straightforward.

\section{REFERENCES}

Abrams, R. A., \& Dobkin, R. S. (1994). Inhibition of return: Effects of attentional cueing on eye movement latencies. Journal of Experimental Psychology: Human Perception \& Performance, 20, 467-477.

Abrams, R. A., \& Pratt, J. (2000). Oculocentric coding of inhibited eye movements to recently attended locations. Journal of Experimental Psychology: Human Perception \& Performance, 12,776-788.

Bennett, P. J., \& Pratt, J. (2001). The spatial distribution of inhibition of return. Psychological Science, 26, 76-80.

Chasteen, A. L., \& Pratt, J. (1999). The effect of inhibition of return on lexical access. Psychological Science, 10, 41-46.

Danzinger, S., Kingstone, A., \& SNYder, J. J. (1998). Inhibition of return to successively stimulated locations in a sequential visual search paradigm. Journal of Experimental Psychology: Human Perception \& Performance, 24, 1467-1475.

Jordan, H., \& TipPer, S. P. (1998). Object-based inhibition of return in static displays. Psychonomic Bulletin \& Review, 5, 504-509.

Kingstone, A., \& Pratt, J. (1999). Inhibition of return is composed of attentional and oculomotor processes. Perception \& Psychophysics, 61, 1046-1054.

KleIN, R, \& TAY LOR, T. L. (1994). Categories of cognitive inhibition with reference to attention. In D. Dagenbach \& T. H. Carr (Eds.), Inhibitory processes in attention, memory, and language (pp. 113-150). San Diego: Academic Press.

MAYLOR, E. (1985). Facilitatory and inhibitory components of orienting in visual space. In M. I. Posner \& O. S. M. Marin (Eds.), Attention and performance XI (pp. 189-204). Hillsdale, NJ: Erlbaum.

Posner, M. I., \& Cohen, Y. (1984). Components of visual orienting. In H. Bouma \& D. G. Bouwhuis (Eds.), Attention and performance $X$ : Control oflanguage processes (pp. 531-556). Hillsdale, NJ: Erlbaum.

Pratt, J., AdAm, J., \& McAuliffe, J. (1998). The spatial relationship between cues and targets mediates inhibition of return. Canadian Journal of Experimental Psychology, 52, 213-216.

Pratt, J., \& McAuliffe, J. (1999). Examining the effect of practice on inhibition of return in static displays. Perception \& Psychophysics, 61, 756-765.

Pratt, J., Spalek, T., \& Bradshaw, E. (1999). The time to detect targets at inhibited and non-inhibited location: Preliminary evidence for attentional momentum. Journal of Experimental Psychology: Human Perception \& Performance, 25, 730-746.

Rafal, R. D., Calabresi, P. A., Brennan, C. W., \& Sciolto, T. K. (1989). Saccade preparation inhibits reorienting to recently attended locations. Journal of Experimental Psychology: Human Perception \& Performance, 15, 673-685.

TAYLOR, T. L., \& KLEIN, R. M. (1998). On the causes and effects of inhibition of return. Psychonomic Bulletin \& Review, 5, 625-643.

Tipper, S. P., Driver, J., \& Weaver, B. (1991). Object-centered inhibition of return of visual attention. Quarterly Journal of Experimental Psychology, 43A, 289-298. 
Tipper, S. P., Jordan, H., \& Weaver, B. (1999). Scene-based and objectcentered inhibition of return: Evidence for dual orienting mechanisms. Perception \& Psychophysics, 61, 50-60.

Tipper, S. P., \& WeAVER, B. (1998). The medium of attention: Locationbased, object-centered or scene-based? In R. Wright (Ed.), Visual attention (pp. 77-107). New York: Oxford University Press.

Tipper, S. P., Weaver, B., Jerreat, L. M., \& BuraK, A. L. (1994). Objectand environment-based inhibition of return of visual attention. Journal of Experimental Psychology: Human Perception \& Performance, 20, 478-499.

Weaver, B., Lupiáñez, J., \& Watson, F. L. (1998). The effects of practice on object-based, location-based, and static-display inhibition of return. Perception \& Psychophysics, 60, 993-1003.

WRIGHT, R, \& RichARD, C. (1996). Inhibition-of-return at multiple locations in visual space. Canadian Journal of Experimental Psychology, 50, 324-327.

YANTIS, S., \& HiLlst Rom, A. P. (1994). Stimulus-driven attentional cap- ture: Evidence from equiluminant visual objects. Journal of Experimental Psychology: Human Perception \& Performance, 20, 95-107.

\section{NOTE}

1. It is unlikely that the participants made eye movements toward the cues or targets in these experiments. When large, very salient cues and targets are used, there is virtually no difference in performance between unmonitored and monitored participants (e.g., Pratt et al., 1999). Moreover, Bennett and Pratt (2001) recently found that such highly salient stimuli are responded to almost equally across a large portion of the visual field. Thus, there is little impetus to move the eyes in such simple detection tasks.

(Manuscript received December 8, 1999; revision accepted for publication January 16, 2001.) 